TAIWANESE JOURNAL OF MATHEMATICS

Vol. 8, No. 4, pp. 559-568, December 2004

This paper is available online at http://www.math.nthu.edu.tw/tjm/

\title{
A GENERAL THEOREM FOR THE GENERALIZED WEYL FRACTIONAL INTEGRAL OPERATOR INVOLVING THE MULTIVARIABLE $H$-FUNCTION
}

\author{
S. P. Goyal and Ritu Goyal
}

\begin{abstract}
In this paper we establish a very general and useful theorem which interconnects the Laplace transform and the generalized Weyl fractional integral operator involving the multivariable $H$-function of related functions of several variables. Our main theorem involves a multidimensional series with essentially arbitrary sequence of complex numbers. By suitably assigning different values to these sequences, one can easily evaluate the generalized Weyl fractional integral operator of special functions of several variables. We have illustrated it for Srivastava-Daoust multivariable hypergeometric function. On account of general nature of this function a number of results involving special functions of one or more variables can be obtained merely by specializing the parameters.
\end{abstract}

\section{INTRODUCTION}

(a) The generalized Weyl fractional integral operator occurring in this paper is defined as follows

(1.1) $=\int_{\nu_{1}}^{\infty} \ldots \int_{\nu_{k}}^{\infty} \prod_{j=1}^{k}\left\{\frac{\left(x_{j}-\nu_{j}\right)^{\mu_{j}-1}}{\Gamma\left(\mu_{j}\right)}\right\} H \begin{gathered}0,0: m_{1}, n_{1} ; \ldots ; m_{k}, n_{k} \\ p, q: p_{1}, q_{1} ; \ldots ; p_{k}, q_{k}\end{gathered}\left[\begin{array}{c}z_{1}\left(x_{1}-\nu_{1}\right)^{-\sigma_{1}} \\ \vdots \\ z_{k}\left(x_{k}-\nu_{k}\right)^{-\sigma_{k}}\end{array}\right]$

$$
W^{\mu_{1}, . ., \mu_{k}}\left\{g\left(x_{1}, \ldots, x_{k} ; u_{1}, \ldots, u_{l}\right) ; \nu_{1}, \ldots, \nu_{k} ; z_{1}, \ldots, z_{k}\right\}
$$$$
. g\left(x_{1}, \ldots, x_{k} ; u_{1}, \ldots, u_{l}\right) d x_{1} \ldots . d x_{k}
$$

provided that the integral on right-hand side of (1.1) converges absolutely.

Received October 17, 2002; revised March 24, 2003.

Communicated by H. M. Srivastava.

2000 Mathematics Subject Classification: Primary 26A33, 33C65; Secondary 44A10.

Key words and phrases: Generalized weyl fractional integral operator, Laplace transform, Parseval-

Goldstein theorem, Fox's $H$-function, Multivariable $H$-function, Srivastava-Daoust multivariable hypergeometric function, Generalized hypergeometric function, Fox-Wright Psi-function. 
In (1.1) and elsewhere $H\left[z_{1}, \ldots, z_{k}\right]$ stands for the multivariable $H$-function introduced by H. M. Srivastava and R. Panda through a series of research papers ([9], [10], [11]). This function is defined and represented in the following manner (see, e.g. [8, p.251. Eq. (C.1)]).

$$
\begin{aligned}
H\left[z_{1}, \ldots, z_{k}\right] & =H_{p, q:\left\{p_{k}, q_{k}\right\}}^{0, n:\left\{m_{k}, n_{k}\right\}}\left[\begin{array}{c|c}
z_{1} & \left(a_{j} ; \alpha_{j}^{\prime}, \ldots, \alpha_{j}^{(k)}\right)_{1, p}:\left\{\left(c_{j}^{(k)}, \gamma_{j}^{(k)}\right)_{1, p_{k}}\right\} \\
\vdots & \left(b_{j} ; \beta_{j}^{\prime}, \ldots, \beta_{j}^{(k)}\right)_{1, q}:\left\{\left(d_{j}^{(k)}, \delta_{j}^{(k)}\right)_{1, q_{k}}\right\}
\end{array}\right] \\
& =\frac{1}{(2 \pi \omega)^{k}} \int_{L_{1}} \ldots \int_{L_{k}} \psi\left(\xi_{1}, \ldots, \xi_{k}\right) \prod_{i=1}^{k}\left(\phi_{i}\left(\xi_{i}\right) z_{i}^{\xi_{i}} d \xi_{i}\right)
\end{aligned}
$$

where $\omega=\sqrt{-1}$, and

$$
\psi\left(\xi_{1}, \ldots, \xi_{k}\right)=\frac{\prod_{j=1}^{n} \Gamma\left(1-a_{j}+\sum_{i=1}^{k} \alpha_{j}^{(i)} \xi_{i}\right)}{\prod_{j=n+1}^{p} \Gamma\left(a_{j}-\sum_{i=1}^{k} \alpha_{j}^{(i)} \xi_{i}\right) \prod_{j=1}^{q} \Gamma\left(1-b_{j}+\sum_{i=1}^{k} \beta_{j}^{(i)} \xi_{i}\right)}
$$

and

$$
\phi_{i}\left(\xi_{i}\right)=\frac{\prod_{j=1}^{m_{i}} \Gamma\left(d_{j}^{(i)}-\delta_{j}^{(i)} \xi_{i}\right) \prod_{j=1}^{n_{i}} \Gamma\left(1-c_{j}^{(i)}+\gamma_{j}^{(i)} \xi_{i}\right)}{\prod_{j=m_{i}+1}^{q_{i}} \Gamma\left(1-d_{j}^{(i)}+\delta_{j}^{(i)} \xi_{i}\right) \prod_{j=n_{i}+1}^{p_{i}} \Gamma\left(c_{j}^{(i)}-\gamma_{j}^{(i)} \xi_{i}\right)} \forall i \in\{1,2, \ldots, k\}
$$

More details of this function can be found in above cited book and research papers. Also $\left\{m_{k}, n_{k}\right\}$ stands for $m_{1}, n_{1} ; \ldots ; m_{k}, n_{k}$ and $\left\{\left(c_{j}^{(k)}, \gamma_{j}^{(k)}\right)_{1, p_{k}}\right\}$ stands for the sequence of $k$ ordered pairs $\left(c_{j}^{\prime}, \gamma_{j}^{\prime}\right)_{1, p_{1}} ; \ldots ;\left(c_{j}^{(k)}, \gamma_{j}^{(k)}\right)_{1, p_{k}}$.

(b) The multivariable hypergeometric function introduced by Srivastava and Daoust [6, p. 454] is defined as follows

$$
\begin{aligned}
& F\left[z_{1}, \ldots, z_{l}\right]=F \begin{array}{l}
t: t_{1} ; \cdots ; t_{l} \\
w: w_{1} ; \cdots ; w_{l}
\end{array}\left[\begin{array}{l}
z_{1} \\
\vdots \\
z_{l}
\end{array}\right] \\
& =F \begin{array}{l}
t:\left\{t_{l}\right\} \\
w:\left\{w_{l}\right\}
\end{array}\left[\begin{array}{l}
\left(g_{j} ; \tau_{j}^{\prime}, \ldots, \tau_{j}^{(l)}\right)_{1, t}:\left\{\left(e_{j}^{(l)}, E_{j}^{(l)}\right)_{1, t_{l}}\right\} ; \\
\left(h_{j} ; \rho_{j}^{\prime}, \ldots, \rho_{j}^{(l)}\right)_{1, w}:\left\{\left(f_{j}^{(l)}, F_{j}^{(l)}\right)_{1, w_{l}}\right\} ;
\end{array} z_{1}, \ldots, z_{l}\right] \\
& =\sum_{r_{1}, \ldots, r_{l}=0}^{\infty} A_{r_{1}, \ldots, r_{l}} \frac{\left(z_{1}\right)^{r_{1}}}{r_{1} !} \ldots \frac{\left(z_{l}\right)^{r_{l}}}{r_{l} !}
\end{aligned}
$$


where

$$
A_{r_{1}, \ldots, r_{l}}=\frac{\prod_{j=1}^{t}\left(g_{j}\right)_{\sum_{i=1}^{l} r_{i} \tau_{j}^{(i)}} \prod_{j=1}^{t_{1}}\left(e_{j}^{\prime}\right)_{r_{1} E_{j}^{\prime}} \ldots \prod_{j=1}^{t_{l}}\left(e_{j}^{(l)}\right)_{r_{l} E_{j}^{(l)}}}{\prod_{j=1}^{w}\left(h_{j}\right)_{\sum_{i=1}^{l} r_{i} \sigma_{j}^{(i)}} \prod_{j=1}^{w_{1}}\left(f_{j}^{\prime}\right)_{r_{1} F_{j}^{\prime}} \ldots \prod_{j=1}^{w_{l}}\left(f_{j}^{(l)}\right)_{r_{l} F_{j}^{(l)}}}
$$

The multiple series (1.5) converges absolutely (see [7]) for all $z_{1}, \ldots, z_{l}$, where $Q_{i}>0$ or for $Q_{i}=0$ and $\left|z_{i}\right|<\rho_{i},(i=1,2, \ldots, l)$ where, $\rho_{i}$ is defined by equation (5.3) in [7, p. 157] and

$$
Q_{i}=1+\sum_{j=1}^{w} \sigma_{j}^{(i)}+\sum_{j=1}^{w_{i}} F_{j}^{(i)}-\sum_{j=1}^{t} \tau_{j}^{(i)}-\sum_{j=1}^{t_{i}} E_{j}^{(i)}, \quad i=1,2, \ldots, l
$$

In this paper we shall establish a theorem which interconnects the well-known multidimensional Laplace transform

$$
\begin{aligned}
\psi\left(s_{1}, \ldots, s_{k}\right)= & L\left\{f\left(x_{1}, \ldots, x_{k}\right) ; s_{1}, \ldots, s_{k}\right\} \\
= & \int_{0}^{\infty} \ldots \int_{0}^{\infty} \exp \left(-\sum_{i=1}^{k} s_{i} x_{i}\right) f\left(x_{1}, . ., x_{k}\right) d x_{1} \ldots d x_{k} \\
& \operatorname{Re}\left(s_{i}\right)>0, i \in\{1,2, \ldots, k\}
\end{aligned}
$$

and generalized Weyl FIO (defined by (1.1)) of related functions. This theorem is then applied to evaluate generalized Weyl FIO of the Srivastava- Daoust multivariable hypergeometric function defined by (1.5). Several (known and new) special cases of our results are mentioned briefly. Our findings may be useful in handling the problems involving Weyl FIO, as our results involve certain special functions that are highly useful in fractional calculus. It may be remarked here that much more general multidimensional integral transformations than (1.8) were considered in [11].

\section{A General Theorem and Its Corollaries}

Theorem. Let $\left\{A_{r_{1}, \ldots, r_{l}}\right\}$ be a sequence of arbitrary complex numbers, $\operatorname{Re}\left(s_{i}\right)>0, \operatorname{Re}\left(\mu_{i}\right)>0, \nu_{i}>0, \nu_{i}^{(j)} \in \mathbf{R}, i \in\{1, \ldots, k\}$ and $j \in\{1, \ldots, l\}$,

$$
h\left(x_{1}, \ldots, x_{k} ; u_{1}, \ldots, u_{l}\right)=\sum_{r_{1}, \ldots, r_{l}=0}^{\infty} A_{r_{1}, \ldots, r_{l}} \prod_{j=1}^{l} \frac{\left(u_{j}\right)^{r_{j}}}{r_{j} !} \prod_{i=1}^{k}\left(x_{i}\right)^{\sum_{j=1}^{l} \nu_{i}^{(j)} r_{j}+\lambda_{i}-1}
$$


and

$$
g\left(s_{1}, \ldots, s_{k} ; u_{1}, \ldots, u_{l}\right)=L\left\{h\left(x_{1}, \ldots, x_{k} ; u_{1}, \ldots, u_{l}\right) ; s_{1}, \ldots, s_{k}\right\}
$$

then

$$
\begin{aligned}
& W^{\mu_{1}, . ., \mu_{k}}\left\{g\left(x_{1}, \ldots, x_{k} ; u_{1}, \ldots, u_{l}\right) ; \nu_{1}, \ldots, \nu_{k} ; z_{1}, \ldots, z_{k}\right\} \\
& =\sum_{r_{1}, \ldots, r_{l}=0}^{\infty} A_{r_{1}, \ldots, r_{l}} \prod_{j=1}^{l} \frac{\left(u_{j}\right)^{r_{j}}}{r_{j} !} \prod_{i=1}^{k}\left\{\frac{1}{\Gamma\left(\mu_{i}\right)}\left(\nu_{i}\right)^{-\left(\sum_{j=1}^{l} \nu_{i}^{(j)} r_{j}+\lambda_{i}\right)+\mu_{i}}\right\} \\
& H \begin{array}{l}
0,0:\left\{m_{k}+1, n_{k}+1\right\} \\
p, q:\left\{p_{k}+1, q_{k}+1\right\}
\end{array} \\
& {\left[\begin{array}{c|l}
z_{1} v_{1}^{-\sigma_{1}} & \left(a_{j} ; \alpha_{j}^{\prime}, \ldots, \alpha_{j}^{(k)}\right)_{1, p}:\left\{\left(1-\lambda_{k}+\mu_{k}-\sum_{j=1}^{l} \nu_{k}^{(j)} r_{j}, \sigma_{k}\right),\left(c_{j}^{(k)}, \gamma_{j}^{(k)}\right)_{1, p_{k}}\right\} \\
\vdots & \\
z_{k} v_{k}^{-\sigma_{k}} & \left(b_{j} ; \beta_{j}^{\prime}, \ldots, \beta_{j}^{(k)}\right)_{1, \mathrm{q}}:\left\{\left(\mu_{k}, \sigma_{k}\right),\left(d_{j}^{(k)}, \delta_{j}^{(k)}\right)_{1, q_{k}}\right\}
\end{array}\right]}
\end{aligned}
$$

and provided that

$\sigma_{i}>0, \operatorname{Re}\left(\mu_{i}\right)-\sigma_{i} \max _{1 \leq j \leq n_{i}} \operatorname{Re}\left\{\left(c_{j}^{(i)}-1\right) / \gamma_{j}^{(i)}\right\}>0, \Omega_{i}>0$, and $\left|\arg z_{i}\right|<\frac{1}{2} \Omega_{i} \pi$, where

$$
\begin{aligned}
\Omega_{i}=-\sum_{j=1}^{p} \alpha_{j}^{(i)}-\sum_{j=1}^{q} \beta_{j}^{(i)}+\sum_{j=1}^{m_{i}} \delta_{j}^{(i)}-\sum_{j=m_{i}+1}^{q_{i}} \delta_{j}^{(i)}+\sum_{j=1}^{n_{i}} \gamma_{j}^{(i)}-\sum_{j=n_{i}+1}^{p_{i}} \gamma_{j}^{(i)} \\
\\
\forall i \in\{1,2, \ldots, k\}
\end{aligned}
$$

and the multiple series on the R.H.S. of (2.1) converges absolutely.

Proof. On substituting the value of $h\left(x_{1}, \ldots, x_{k} ; u_{1}, \ldots, u_{l}\right)$ from (2.1) in (2.2) and evaluating the multidimensional Laplace transform, we find that

$$
\begin{aligned}
& g\left(\mathrm{~s}_{1}, . ., \mathrm{s}_{k} ; u_{1}, \ldots, u_{l}\right) \\
& \quad=\sum_{r_{\hat{1}}, \ldots, r_{l}=0}^{\infty} A_{r_{\hat{1}}, \ldots, r_{\hat{l}}} \prod_{j=1}^{l} \frac{\left(u_{j}\right)^{r_{j}}}{r_{j} !} \prod_{i=1}^{k}\left(s_{i}\right)^{-\sum_{j=1}^{l} \nu_{i}^{(j)} r_{j}-\lambda_{i}} \Gamma\left(\lambda_{i}+\sum_{j=1}^{l} \nu_{i}^{(j)} r_{j}\right)
\end{aligned}
$$

where $\operatorname{Re}\left(s_{i}\right)>0, \operatorname{Re}\left(\lambda_{i}\right)>0, \nu_{i}^{(j)} \in \mathbf{R}, i \in\{1, \ldots, k\}, j \in\{1, \ldots, l\}$ and multiple series on R.H.S. of (2.5) is absolutely convergent.

Again taking the multidimensional Laplace transform of the multivariable $\mathrm{H}$ function and replacing $x_{\hat{i}}$ by $\left(x_{\hat{i}}-\nu_{\hat{i}}\right)$ and using first shift rule therein, we get (2.6)

$$
\begin{aligned}
& L\left\{\prod_{i=1}^{k}\left(\left(x_{i}-\nu_{i}\right)^{\mu_{i}-1} H\left(x_{i}-\nu_{i}\right)\right) H_{1}\left[z_{1}\left(x_{1}-\nu_{1}\right)^{-\sigma_{1}}, \ldots, z_{k}\left(x_{k}-\nu_{k}\right)^{-\sigma_{k}}\right] ; s_{1}, \ldots, s_{k}\right\} \\
& \quad=\exp \left\{-\sum_{i=1}^{k} \nu_{i} s_{i}\right\} \psi\left(s_{1}, \ldots, s_{k}\right)
\end{aligned}
$$


where $H(x-\nu)$ is the well-known Heaviside Unit function and $H_{1}\left[z_{1}, \ldots, z_{k}\right] \equiv$ $\left\{H\left[z_{1}, \ldots, z_{k}\right]\right\}_{n=0}$ Also,

$$
\begin{aligned}
\psi\left(s_{1}, . ., s_{k}\right)= & \prod_{i=1}^{k}\left(s_{i}^{-\mu_{i}}\right) H \begin{array}{l}
0,0:\left\{m_{k}+1, n_{k}\right\} \\
p, q:\left\{p_{k}, q_{k}+1\right\}
\end{array} \\
& {\left[\begin{array}{c|l}
z_{1} s_{1}^{\sigma_{1}} & \left(a_{j} ; \alpha_{j}^{\prime}, \ldots, \alpha_{j}^{(k)}\right)_{1, p}:\left\{\left(c_{j}^{(k)}, \gamma_{j}^{(k)}\right)_{1, p_{k}}\right\} \\
\vdots & \left(b_{j} ; \beta_{j}^{\prime}, \ldots, \beta_{j}^{(k)}\right)_{1, \mathrm{q}}:\left\{\left(\mu_{k}, \sigma_{k}\right),\left(d_{j}^{(k)}, \delta_{j}^{(k)}\right)_{1, q_{k}}\right\}
\end{array}\right] }
\end{aligned}
$$

The sufficient condition of validity for (2.6) are

$$
\sigma_{i}>0, \operatorname{Re}\left(s_{i}\right)>0,\left|\arg z_{i}\right|<\frac{1}{2} \Omega_{i} \pi, \Omega_{i}>0,
$$

and $\operatorname{Re}\left(\mu_{i}\right)-\sigma_{i} \max _{1 \leq j \leq n} \operatorname{Re}\left\{\left(c_{j}^{(i)}-1\right) / \gamma_{j}^{(i)}\right\}>0 \forall i \in\{1, \ldots, k\}$

Now applying the multidimensional analogue of the Parseval-Goldstein theorem for the Laplace transform for the pairs (2.2) and (2.6), we have

$$
\begin{aligned}
\text { L.H.S. of }(2.3)= & \int_{0}^{\infty} \ldots \int_{0}^{\infty} \exp \left\{-\sum_{i=1}^{k} \nu_{i} x_{i}\right\} \psi\left(x_{1}, \ldots, x_{k}\right) \\
& h\left(x_{1}, . x_{k} ; u_{1}, \ldots, u_{l}\right) d x_{1} \ldots d x_{k}
\end{aligned}
$$

Substituting the series form of $h\left(x_{1}, \ldots, x_{k} ; u_{1}, \ldots, u_{1}\right)$ from $(2.1), \psi\left(x_{1}, \ldots, x_{k}\right)$ from (2.7) in the R.H.S. of (2.8) and interchanging the order of integration and summation therein and then evaluating the $\mathrm{x}$-integral thus obtained, we arrive easily at the required result (2.3).

(i) Taking $l=k$ and choosing, $\nu_{i}^{(j)}=0, i \neq j$ and $\nu_{i}^{(i)}=\nu_{i}$ in our main theorem, we immidiately get

Corollary 1. Let $\left\{A_{r_{1}, \ldots, r_{k}}\right\}$ be a sequence of arbitrary complex numbers,

$$
h\left(x_{1}, \ldots, x_{k} ; u_{1}, \ldots, u_{k}\right)=\sum_{r_{1}, \ldots, r_{k}=0}^{\infty} A_{r_{1}, \ldots, r_{k}} \prod_{i=1}^{k}\left(\frac{\left(u_{i}\right)^{r_{i}}}{r_{i} !}\left(x_{i}\right)^{\nu_{i} r_{i}+\lambda_{i}-1}\right)
$$

and

$$
g\left(s_{1}, \ldots, s_{k} ; u_{1}, \ldots, u_{k}\right)=L\left\{h\left(x_{1}, . ., x_{k} ; u_{1}, \ldots, u_{k}\right) ; s_{1}, \ldots, s_{k}\right\}
$$

then

$$
\begin{aligned}
& W^{\mu_{1}, . ., \mu_{k}}\left\{g\left(x_{1}, \ldots, x_{k} ; u_{1}, \ldots, u_{k}\right) ; \nu_{1}, \ldots, \nu_{k} ; z_{1}, \ldots, z_{k}\right\} \\
& =\sum_{r_{1}, \ldots, r_{k}=0}^{\infty} A_{r_{1}, \ldots, r_{k}} \prod_{i=1}^{k} \frac{\left(u_{i}\right)^{r_{i}}}{r_{i} ! \Gamma\left(\mu_{i}\right)}\left(\nu_{i}\right)^{-\nu_{i} r_{i}-\lambda_{i}+\mu_{i}} H \begin{array}{c}
0,0:\left\{m_{k}+1, n_{k}+1\right\} \\
p, q:\left\{p_{k}+1, q_{k}+1\right\}
\end{array} \\
& {\left[\begin{array}{c}
z_{1} v_{1}^{-\sigma_{1}} \\
\vdots \\
z_{k} v_{k}^{-\sigma_{k}}
\end{array} \mid \begin{array}{l}
\left(a_{j} ; \alpha_{j}^{\prime}, \ldots, \alpha_{j}^{(k)}\right)_{1, p}:\left\{\left(1-\lambda_{k}+\mu_{k}-\nu_{k} r_{k}, \ldots, \sigma_{k}\right),\left(c_{j}^{(k)}, \gamma_{j}^{(k)}\right)_{1, \mathrm{q}}:\left\{\left(\mu_{k}, \sigma_{k}\right),\left(d_{j}^{(k)}, \delta_{j}^{(k)}\right)_{1, q_{k}}\right\}\right.
\end{array}\right]}
\end{aligned}
$$


provided that the conditions (modified appropriately) given with the main theorem are satisfied.

(ii) Letting $p=q=0, m_{i}=1, n_{i}=p_{i}, q_{i} \rightarrow q_{i}+1, \sigma_{i}=1 \forall i=1,2, . ., k$, all $\gamma$ 's and $\delta$ 's equal to unity in (2.3) and using the known result [8, p.18, Eq. (2.6.3)] therein, we have after a little simplification

\section{Corollary 2.}

$$
\begin{aligned}
W_{1}^{\mu_{1}, . ., \mu_{k}}\left\{g\left(x_{1}, \ldots, x_{k} ; u_{1}, \ldots, u_{l}\right) ; \nu_{1}, \ldots, \nu_{k} ; z_{1}, \ldots, z_{k}\right\} \\
=\int_{\nu_{1}}^{\infty} \ldots \int_{\nu_{k}}^{\infty} \prod_{i=1}^{k}\left\{\frac{\left(x_{i}-\nu_{i}\right)^{\mu_{i}-1}}{\Gamma\left(\mu_{i}\right)} p_{i} F_{q_{i}}\left[\left(c_{p_{i}}\right) ;\left(d_{q_{i}}\right) ; \frac{-z_{i}}{x_{i}-v_{i}}\right]\right\} \\
\left.=\prod_{i=1}^{k}\left\{\frac{v_{i}^{\mu_{i}-\lambda_{i}}}{\Gamma\left(\mu_{i}\right)} \prod_{j=1}^{q_{i}} \Gamma\left(x_{k} ; u_{1}, \ldots, u_{l}\right) d x_{1} \ldots d x_{k}\right)\left\{\prod_{j=1}^{p_{i}} \Gamma\left(c_{j}^{(i)}\right)\right\}^{-1}\right\} \sum_{r_{1}, \ldots, r_{l}=0}^{\infty} A_{r_{1}, \ldots, r_{l}} \prod_{j=1}^{l}\left(\frac{\left(u_{j}\right)^{r_{j}}}{r_{j} !}\right) \\
\cdot \prod_{i=1}^{k}\left(\nu_{i}\right)^{-\sum_{j=1}^{l} \nu_{i}^{(j)} r_{j}} G_{p_{i}+1, q_{i}+2}^{2, p_{i}+1}\left[\frac{z_{i}}{\nu_{i}} \mid \begin{array}{l}
\left.1-\lambda_{i}+\mu_{i}-\sum_{j=1}^{l} \nu_{i}^{(j)} r_{j},\left(1-c_{p_{i}}^{(i)}\right)\right] \\
0, \mu_{i},\left(1-d_{q_{i}}^{(i)}\right)
\end{array}\right.
\end{aligned}
$$

where $h\left(x_{1}, \ldots x_{k} ; u_{1}, \ldots, u_{l}\right)$ and $g\left(x_{1}, \ldots x_{k} ; u_{1},, \ldots, u_{l}\right)$ are given by $(2.1)$ and (2.2) respectively. The conditions of validity for (2.12) are

(a) $\nu_{i}>0, \operatorname{Re}\left(\mu_{i}\right)>0$ and $\nu_{i}^{(j)} \in \mathrm{R},(j=1,2, \ldots, l)$

(b) $p_{i} \leq q_{i}$ or $p_{i}=q_{i}+1$ with $\left|z_{i}\right|<1, i \in\{1, \ldots, k\}$

(c) the multiple series on the R.H.S. of (2.12) converges absolutely.

(iii) Further, taking $p_{i}=1, q_{i}=0, z_{i}=v_{i}$ and replacing $\mu_{i}+c_{i}$ by $\mu_{i}(\mathrm{i}=1, \ldots, \mathrm{k})$ in Corollary 2 and using known results [5, p. 74, Eq. (1); 8, p. 91, Eq. (6.4.19)] we arrive at the following interesting result contained in

\section{Corollary 3.}

$$
\begin{aligned}
& \mathrm{W}_{2}^{\mu_{1}, \ldots, \mu_{k}}\left[g\left(x_{1}, . ., x_{k} ; u_{1}, \ldots, u_{l}\right) ; \nu_{1}, \ldots, \nu_{k}\right] \\
& =\int_{v_{1}}^{\infty} \ldots \int_{v_{k}}^{\infty} \prod_{i=1}^{k}\left\{\frac{\left(x_{i}-\nu_{i}\right)^{\mu_{i}-1}}{\Gamma\left(\mu_{i}\right)} x_{i}^{-c_{i}}\right\} g\left(x_{1}, . ., x_{k} ; u_{1}, \ldots, u_{l}\right) d x_{1} \ldots . d x_{k} \\
& =\sum_{r_{1}, \ldots, r_{l}=0}^{\infty} A_{r_{1}, \ldots, r_{l}} \prod_{j=1}^{l}\left(\frac { ( u _ { j } ) ^ { r _ { j } } } { r _ { j } ! } \prod _ { i = 1 } ^ { k } \left[\left(\nu_{i}\right)^{-\sum_{j=1}^{l} \nu_{i}^{(j)} r_{j}+\mu_{i}-c_{i}-\lambda_{i}} \Gamma\left(\lambda_{i}+\sum_{j=1}^{l} \nu_{i}^{(j)} r_{j}\right)\right.\right. \\
& \left.\quad . \Gamma\left(\lambda_{i}-\mu_{i}+c_{i}+\sum_{j=1}^{l} \nu_{i}^{(j)} r_{j}\right)\left(\Gamma\left(\lambda_{i}+c_{i}+\sum_{j=1}^{l} \nu_{i}^{(j)} r_{j}\right)\right)^{-1}\right]
\end{aligned}
$$


If we take $k=l$ and $\nu_{i}^{(j)}=0$ for $i \neq j$ and $\nu_{i}^{(i)}=\nu_{i}$ in Corollary 3, we get result due to Goyal and Garg [3, p.274, Theorem 2]. Again taking $l=2, k=1$, $\nu_{1}=\nu_{2}=1$ in (2.13), we arrive at the known Theorem 3 due to Jain and Pathan [4, p.53, Eq. (2.9)] and for $l=2, k=1, \nu_{1}=0, \nu_{2}=1$, the Corollary 3 reduces to Theorems 1 and 2 due to them. Also for $\mathrm{k}=1$, the main Theorem reduces to a result recently given by the authors [2].

\section{Further CONSEQuences AND APPLications}

If we choose $\left\{A_{r_{1}, \ldots, r_{l}}\right\}$ as given by (1.6), then from (1.5), (2.1) and (2.2) we have,

$$
\begin{aligned}
& g\left(s_{1}, . ., s_{k} ; u_{1}, \ldots, u_{l}\right) \\
& =\prod_{i=1}^{k}\left(\Gamma\left(\lambda_{i}\right) s_{i}^{-\lambda_{i}}\right) F_{1}\left[u_{1} \prod_{i=1}^{k}\left(s_{i}^{-\nu_{i}^{\prime}}\right), \ldots, u_{l} \prod_{i=1}^{k}\left(s_{i}^{-\nu_{i}^{(l)}}\right)\right]
\end{aligned}
$$

where

$$
\begin{aligned}
& F_{1}\left[u_{1}, \ldots, u_{l}\right]=F_{w:\left\{w_{l}\right\}}^{t+k:\left\{t_{l}\right\}} \\
& {\left[\begin{array}{ll}
\left(\lambda_{i} ; \nu_{1}^{(i)}, \ldots, \nu_{l}^{(i)}\right)_{1, k},\left(g_{j} ; \tau_{j}^{\prime}, \ldots, \tau_{j}^{(l)}\right)_{1, t} & \left.:\left\{\left(e_{j}^{(l)}, E_{j}^{(l)}\right)_{1, t_{l}}\right\} ; u_{1}, \ldots, u_{l}\right] \\
\left(h_{j} ; \rho_{j}^{\prime}, \ldots, \rho_{j}^{(l)}\right)_{1, w} & :\left\{\left(f_{j}^{(l)}, F_{j}^{(l)}\right)_{1, w_{l}}\right\} ;
\end{array}\right]}
\end{aligned}
$$

provided that $\operatorname{Re}\left(s_{i}\right)>0, \operatorname{Re}\left(\lambda_{i}\right)>0, \nu_{i}^{(j)} \in \mathbf{R}^{+},(i=1, \ldots, k ; j=1, \ldots, l)$ and the conditions mentioned with (1.6) are satisfied.

Substituting the value of $\left\{A_{r_{1}, \ldots, r_{l}}\right\}$ and $g\left(x_{1}, \ldots x_{k} ; u_{1},, \ldots, u_{l}\right)$ in (2.3) of the main theorem, then using (1.2), (1.5) and [8, p. 254, Eq. (C.9)] therein and reinterpreting the expression so obtained in terms of $\mathrm{H}$-Function of $(\mathrm{k}+l)$ variables, we finally obtain

$$
\begin{aligned}
& W^{\mu_{1}, \ldots, \mu_{k}}\left\{\prod_{i=1}^{k}\left(x_{i}^{-\lambda_{i}}\right) F_{1}\left[u_{1} \prod_{i=1}^{k}\left(x_{i}^{-\nu_{i}^{\prime}}\right), \ldots, u_{l} \prod_{i=1}^{k}\left(x_{i}^{-\nu_{i}^{(l)}}\right)\right] ; \nu_{1}, \ldots, \nu_{k} ; z_{1}, . ., z_{k}\right\} \\
& =\prod_{i=1}^{k}\left(\frac{\nu_{i}^{\mu_{i}-\lambda_{i}}}{\Gamma\left(\mu_{i}\right) \Gamma\left(\lambda_{i}\right)}\right) \prod_{j=1}^{w} \Gamma\left(h_{j}\right)\left\{\prod_{j=1}^{t} \Gamma\left(g_{j}\right)\right\}^{-1} \prod_{i=1}^{l}\left[\prod_{j=1}^{w_{i}} \Gamma\left(f_{j}^{(i)}\right)\left\{\prod_{j=1}^{t_{i}} \Gamma\left(e_{j}^{(i)}\right)\right\}^{-1}\right]
\end{aligned}
$$




$$
\begin{aligned}
& H \begin{array}{l}
0, t+k:\left\{m_{k}+1, n_{k}\right\} ;\left\{1, t_{l}\right\} \\
p+t+k, w+q:\left\{p_{k}, q_{k}+1\right\} ;\left\{t_{l}, w_{l}+1\right\}
\end{array}\left[\begin{array}{c}
z_{1} \nu_{1}^{-\sigma_{1}} \\
\vdots \\
z_{k} \nu_{k}^{-\sigma_{k}} \\
-u_{1} \prod_{i=1}^{k}\left(\nu_{i}^{-\nu_{i}^{\prime}}\right) \\
\vdots \\
-u_{l} \prod_{i=1}^{k}\left(\nu_{i}^{-\nu_{i}^{(l)}}\right)
\end{array}\right) \\
& \cdot\left(1-\lambda_{j}+\mu_{j} ; 0, . ., \sigma_{j}, \ldots 0, \nu_{j}^{\prime}, \ldots, \nu_{j}^{(l)}\right)_{1, k}, \\
& (1-g_{j} ; \underbrace{0, \ldots, 0}_{k-\text { times }}, \tau_{j}^{\prime}, \ldots, \tau_{j}^{(l)})_{1, t},(a_{j} ; \alpha_{j}^{\prime}, \ldots, \alpha_{j}^{(k)}, \underbrace{0, \ldots, 0}_{l-\text { times }})_{1, p}:\left\{\left(c_{j}^{(k)}, \gamma_{j}^{(k)}\right)_{1, p_{k}}\right\} ; \\
& (1-h_{j} ; \underbrace{0, \ldots, 0}_{k-\text { times }}, \rho_{j}^{\prime}, \ldots, \rho_{j}^{(l)})_{1, w},(b_{j} ; \beta_{j}^{\prime}, \ldots, \beta_{j}^{(k)}, \underbrace{0, \ldots, 0}_{l-\text { times }}):\left\{\left(\mu_{k}, \sigma_{k}\right),\left(d_{j}^{(k)}, \delta_{j}^{(k)}\right)_{1, q_{k}}\right\} ; \\
& \left\{\left(1-e_{j}^{(l)}, E_{j}^{(l)}\right)_{1, t_{l}}\right\} \\
& \left.\left\{(0,1),\left(1-f_{j}^{(l)}, F_{j}^{(l)}\right)_{1, w_{l}}\right\}\right]
\end{aligned}
$$

The conditions of validity of (3.3) are

(i) $\nu_{i}>0, \sigma_{i}>0, \nu_{i}^{(j)} \in \mathbf{R}^{+}, i \in\{1,2, \ldots, k\}, j \in\{1,2, \ldots, l\}$

$$
\begin{aligned}
& \operatorname{Re}\left(\lambda_{i}\right)+\sigma_{i} \min _{1 \leq j \leq m_{i}} \operatorname{Re}\left(d_{j} / \delta_{j}\right)>1 \text { and } \\
& \operatorname{Re}\left(\mu_{i}\right)-\sigma_{i} \max _{1 \leq j \leq n_{i}} \operatorname{Re}\left\{\left(c_{j}-1\right) / \gamma_{j}\right\}>0,
\end{aligned}
$$

(ii) $\Omega_{i}>0,\left|\arg z_{i}\right|<\frac{1}{2} \Omega_{i} \pi$, where $\Omega_{i}$ is defined by (2.4)

$$
\forall i \in\{1,2, \ldots, k\}
$$

(iii) the conditions (modified appropriately) which are given just below (1.6).

Next we mention certain interesting special cases of the aforementioned result by considering following examples:

(i) If we let $l=1$ and $t=w=0$ in (3.3) and use a known result (c.f., e.g. [8, p. 19, Eq. (2.6.11)]) therein, we obtain the following result involving the Fox-Wright Psi- function. 


$$
\begin{aligned}
& W^{\mu_{1}, \ldots, \mu_{k}}\left\{\prod _ { i = 1 } ^ { k } x _ { i + k } ^ { - \lambda _ { i } } \psi _ { w } \left[\begin{array}{l}
\left(\lambda_{j}, \nu_{j}\right)_{1, k},\left(e_{j}, E_{j}\right)_{1, t} ; \prod_{i=1}^{k} x_{i}^{-\nu_{i}} \\
\left.\left(f_{j}, F_{j}\right)_{1, w} ; \nu_{1}, \ldots, \nu_{k} ; z_{1}, \ldots, z_{k}\right\}
\end{array}\right.\right. \\
& =\prod_{i=1}^{k}\left\{\frac{\nu_{i}^{\mu_{i}-\lambda_{i}}}{\Gamma\left(\mu_{i}\right)}\right\} H \begin{array}{l}
0, k:\left\{m_{k}+1, n_{k}\right\} ; 1, t \\
p+k, q:\left\{p_{k}, q_{k}+1\right\} ; t, w+1
\end{array}\left[\begin{array}{c}
z_{1} \nu_{1}^{-\sigma_{1}} \\
\vdots \\
z_{k} \nu_{k}^{-\sigma_{k}} \\
-u \prod_{i=1}^{k}\left(\nu_{i}^{-\nu_{i}}\right)
\end{array} \mid\right. \\
& \left(1-\lambda_{j}+\mu_{j} ; 0, . ., \sigma_{j}, \ldots 0, \nu_{j}\right)_{1, k} \\
& (a_{j} ; \alpha_{j}^{\prime}, \ldots, \alpha_{j}^{(k)}, \underbrace{0, \ldots, 0}_{l-\text { times }})_{1, p}:\left\{\left(c_{j}^{(k)}, \gamma_{j}^{(k)}\right)_{1, p_{k}}\right\} ;\left(1-e_{j}, E_{j}\right)_{1, t} \\
& (b_{j} ; \beta_{j}^{\prime}, \ldots, \beta_{j}^{(k)}, \underbrace{0, . ., 0}_{l-\text { times }})_{1, \mathrm{q}}:\left\{\left(\mu_{k}, \sigma_{k}\right),\left(d_{j}^{(k)}, \delta_{j}^{(k)}{ }_{1, q_{k}}\right\} ;(0,1),\left(1-f_{j}, F_{j}\right)_{1, w}\right]
\end{aligned}
$$

(ii) If we substitute $\left\{A_{r_{1}, \ldots, r_{l}}\right\}$ from (1.6) in (2.13), we obtain

$$
\begin{aligned}
W_{2}^{\mu_{1}, \ldots, \mu_{k}}\left\{\prod_{i=1}^{k}\left(x_{i}^{-\lambda_{i}}\right) F_{1}\left[u_{1} \prod_{i=1}^{k}\left(x_{i}^{-\nu_{i}^{\prime}}\right), \ldots, u_{l} \prod_{i=1}^{k}\left(x_{i}^{-\nu_{i}^{(l)}}\right)\right] ; \nu_{1}, \ldots, \nu_{k}\right\} \\
\left.=\prod_{i=1}^{k}\left\{\frac{\nu_{i}^{\mu_{i}-c_{i}-\lambda_{i}} \Gamma\left(\lambda_{i}-\mu_{i}+c_{i}\right)}{\Gamma\left(\lambda_{i}+c_{i}\right)}\right\} \begin{array}{l}
t+2 k:\left\{t_{k}\right\} \\
\begin{array}{r}
t \\
\mathrm{w}+k:\left\{w_{k}\right\}
\end{array}\left[\begin{array}{l}
\left(\lambda_{j}-\mu_{j}+\mathrm{c}_{j} ; \nu_{j}^{\prime}, \ldots, \nu_{j}^{(l)}\right)_{1, k}, \\
\left(\lambda_{j}+c_{j} ; \nu_{j}^{\prime}, \ldots, \nu_{j}^{(l)}\right)_{1, k}
\end{array}\right. \\
\left(\lambda_{j} ; \nu_{j}^{\prime}, \ldots, \nu_{j}^{(l)}\right)_{1, k},\left(g_{j} ; \tau_{j}^{\prime}, \ldots, \tau_{j}^{(l)}\right)_{1, t}:\left\{\begin{array}{l}
\left.\left(e_{j}^{(l)}, E_{j}^{(l)}\right)_{1, t_{l}}\right\} \\
\left(h_{j} ; \rho_{j}^{\prime}, \ldots, \rho_{j}^{(l)}\right)_{1, w}:\left\{\begin{array}{l}
\left(f_{j}^{(l)}, F_{j}^{(l)}\right)_{1, w_{l}}
\end{array}\right\} ;
\end{array}\right. \\
u_{1} \prod_{i=1}^{k}\left(\nu_{i}^{-\nu_{i}^{\prime}}\right), \ldots, u_{k} \prod_{i=1}^{k}\left(\nu_{i}^{-\nu_{i}^{(l)}}\right)
\end{array}\right]
\end{aligned}
$$

The conditions of validity for results (3.4) and (3.5) are easily obtainable from the main result (3.3), however we prefer to omit them.

(ii) Also on taking $k=1, l=2$ in (3.5) we arrive at a known result due to Jain and Pathan [4, p.54, Eq. (3.4)], which also contains another two results (3.6) and (3.8) due to them. Finally on taking $l=k, \nu_{i}^{(j)}=0$ for $i \neq j$ and $\nu_{i}^{(i)}=\nu_{i}$, we get the result (4.5) due to Goyal and Garg [3] as a special case of our result (3.5). 


\section{ACKNOWLEDGEMENT}

The authors are thankful to Professor H. M. Srivastava (University of Victoria, Canada) for his generous help and very useful suggestions during the preparation of this paper. The second author is also thankful to the Council of Scientific and Industrial Research, India for awarding Junior Research Fellowship, under the research scheme No. 9/149(294)2K2/EMR-I.

\section{REFERENCES}

1. A. Erdelyi, et al., Tables of Integral Transforms, Vol. 1, McGraw-Hill, New York, 1954.

2. S. P. Goyal and Goyal Ritu, A general theorem involving generalized (Weyl) fractional integral operator, Aligarh Bull. Math., 21 (2002), 45-55.

3. S. P. Goyal and Rashmi Garg, On Laplace transform and Weyl fractional integral operator for one or more variables, Far East J. Math. Sci., 7 (2002), 269-283.

4. R. Jain and M. A.Pathan, On Theorems involving Laplace Transform and Weyl Fractional Integral Operator, Proceedings of Second International Conference of Society for Special Functions and their Applications, (eds. R. Y. Denis, and M. A. Pathan), II (2001), 51-56.

5. E. D. Rainville, Special Functions, Chelsea Publ. Comp. Bronx, New York, 1971.

6. H. M. Srivastava and M. C. Daoust, Certain generalized Neumann expansions associated with the Kampé de Feriet function, Nederl. Akad. Wetensch. Proc. Ser., A 72 (1969), 449-457.

7. H. M. Srivastava and M. C. Daoust, A note on the convergence of Kampé de Fériet double hypergeometric series, Math. Nachr., 53 (1972), 151-157.

8. H. M. Srivastava, K. C. Gupta and S. P. Goyal, The H-Functions of One and Two Variables with Applications, South Asian Publishers, New Delhi and Madras, 1982.

9. H. M. Srivastava and R. Panda, Some expansion theorems and generating relations for the H-function of several complex variables I and II, Comment. Math. Univ. St. Paul. 24 (1975), 119-137; ibid, 25 (1976), 167-197.

10. Srivastava H. M. and Panda R., Some bilateral generating functions for a class of generalized hypergeometric polynomials, J. Reine Angew. Math., 283/284 (1976), 265-274.

11. H. M. Srivastava and R. Panda, Certain multidimensional integral transformations I and II, Nederl. Akad. Wetensch. Proc. Ser., A81=Indag. Math., 40 (1978), 118-131 and $132-144$.

S. P. Goyal and Ritu Goyal

Department of Mathematics,

University of Rajasthan,

Jaipur 302 004, Rajasthan,

India 